\title{
Visualization of High-Speed Impact of Penetrator into Icy Target*
}

\author{
Kojiro Suzuki', Kazuya Namba², Yasumasa Watanabe ${ }^{2}$ \\ ${ }^{1}$ Department of Advanced Energy, Graduate School of Frontier Sciences, The University of Tokyo, Tokyo, Japan \\ ${ }^{2}$ Department of Aeronautics and Astronautics, Graduate School of Engineering, The University of Tokyo, Tokyo, \\ Japan \\ Email: kjsuzuki@k.u-tokyo.ac.jp
}

Received 30 January 2016; accepted 15 March 2016; published 29 April 2016

Copyright (C) 2016 by authors and Scientific Research Publishing Inc.

This work is licensed under the Creative Commons Attribution International License (CC BY).

http://creativecommons.org/licenses/by/4.0/

(c) (i) Open Access

\section{Abstract}

For application to exploration under the surface of icy objects in the solar system, the penetration of an impact probe into an icy target was experimentally simulated by using the ballistic range. Slender projectiles with a cylindrical body and various nose shapes were tested at the impact velocity $130-420 \mathrm{~m} / \mathrm{s}$. The motion of the penetrator, fragmentation of ice and crater forming were observed by the high-speed camera. It revealed that the crown-shaped ejection was made for a short time after the impact and then the outward normal jet-like stream of ice pieces continued for much longer time. The concave shape of the crater was successfully visualized by pouring the plaster into it. The two-stage structure, the pit and the spall, was clearly confirmed. The rim was not formed around the crater. Observation of the crater surface and the ice around the trace of the penetrator shows that both crushing into smaller ice pieces and recompression into ice blocks are caused by the forward motion of the penetrator. In case of a body with a flow-through duct, ice pieces entering the inlet at the nose tip were ejected from the tail, resulting in relaxation of the impact force. The correlation of the penetration distance and the crater diameter with the impact velocity was investigated.

\section{Keywords}

Penetrator, Ice, Impact, Crater Forming, Ballistic Range, High-Speed Camera

\section{Introduction}

Recently the presence of water ice has been found at various places in the solar system. For example, the nuc"Presented at The 13th International Symposium on Fluid Control, Measurement and Visualization (FLUCOME2015), 15-18 November 2015, Doha, Qatar. 
leus of a comet is known to be mainly composed of ice. Such icy objects have been attracting our attention as a target of exploration, because the presence of water is believed to be one of the necessary conditions for formation of life in the universe [1].

For exploration under the surface of an object in the solar system, we must send a probe, which lands on the surface after the interplanetary cruise, and puts scientific instruments at an appropriate depth under the surface by using some device like a drill. However, such mission scenario will require a relatively complicated system and large mass for both the spacecraft and the fuel for the trajectory maneuvering. Consequently, it is not suitable for a low-cost mission.

On the other hand, an impact probe called "penetrator" seems promising [2]. A penetrator directly hits the surface of a target object and goes into its interior without making a "soft" landing. It is an intelligent impact probe, which includes scientific instruments, data recorders, power supply, communication system and all other necessary equipments in it. Such simplicity of the mission scenario is favorable from a viewpoint of the recent trend of small and low-cost mission, because it efficiently utilizes the kinetic energy of the orbital or interplanetary flight for penetration. In Japan, a penetrator mission called "LUNAR-A" to the moon was considered by the Japan Aerospace Exploration Agency (JAXA). Though the mission was cancelled due to the delay in the spacecraft development, the design of the penetrator body was successfully completed based on a large amount of the experiments simulating the penetration into the lunar regolith [3]. The case of the LUNAR-A mission tells us both the fascination of the penetrator mission, and the difficulty in understanding the penetration dynamics and appropriately designing the penetrator body, which can survive under the severe impact force with thousands of deceleration G's.

The advantage of the penetrator is also expected for the mission to icy objects in the solar system. In fact, the concept study on the comet nucleus penetrator mission, CRAF (Comet Rendezvous and Asteroid Flyby), was made by NASA in 1980's [2]. We cannot design the ice penetrator without clear understanding of the penetration dynamics into icy target. However, our knowledge obtained on it so far is quite limited. The crater forming on the icy surface has been mainly studied in the field of the planetary science [4]-[6]. In [4] [5], the crater forming experiments on the water ice were carried out at the impact velocity up to about $800 \mathrm{~m} / \mathrm{s}$ using the gas gun. Based on the observation of the cross section, the shape of the crater was classified into seven types in [4]. The scaling laws were found on the relation among the impact energy, crater size, ejecta mass, and so on [4] [5]. The shock wave propagating in the icy target was experimentally investigated at high impact velocities from 3 $\mathrm{km} / \mathrm{s}$ to $3.8 \mathrm{~km} / \mathrm{s}$ by using the two-stage light gas gun in [6]. In the above studies, the properties of the crater after the impact were focused rather than the transient process of its formation. The dynamic behavior of the fragmented ice pieces, in other words, the flow of ice pieces around the penetrator, during the penetration has not been clarified yet. In addition, most of the experiments have been made for impactors with small length-to-diameter ratio like a sphere in order to simulate natural objects in the solar system. No experimental results are available for slender bodies like penetrators. To determine appropriately the shape, the location of the center of gravity, the body structure, the impact conditions and so on, the prediction method for the force acting on the body and the penetration trajectory must be established based on the penetration experiments of slender bodies.

In the present study, we investigated the fundamental characteristics of the penetration dynamics into the water ice by conducting the ballistic range experiments for cone-cylinder projectiles of the mass about $2.0-2.5 \mathrm{~g}$ at the impact velocity from $130 \mathrm{~m} / \mathrm{s}$ to $420 \mathrm{~m} / \mathrm{s}$. To enrich our knowledge about the penetration dynamics into the icy target, visualization is apparently the most powerful tool. The images of the high-speed video camera revealed both the motion of the penetrator and the behavior of fragmented ice pieces with high temporal resolution. In addition, the detailed observation of the crater shape after the experiment gave us a hint to understand the trajectory of the penetrator in the ice.

The objectives of the present study are: 1) to clarify the process of fragmentation and crater forming of the icy target by the penetrator from the images by the high-speed video camera; 2 ) to visualize the shape of the crater made by the penetrator and 3) to find the characteristic features of the dynamics of the penetration and crater forming in the case of an icy target.

\section{Ballistic Range and Experimental Models}

In the present study, the ballistic range in the authors' laboratory was used to simulate the impact of the penetrator at an icy target. The ballistic range consists of the high-pressure chamber, shock tube, acceleration tube 
and the test chamber, as shown in Figure 1. The detail of the test chamber is shown in Figure 2. The penetrator model attached to the sabot was loaded at the connection between the shock tube and the acceleration tube. The sabot was made from the ABS resin and its mass was 3.0 - 3.5 g. The test section was open to the atmosphere and all the experiments were conducted under the atmospheric pressure. However, to reduce the velocity loss due to the aerodynamic drag force acting on the front surface of the penetrator and the sabot, the acceleration tube was sealed by setting thin film (diaphragm) at both ends and was evacuated down to about $1 \mathrm{kPa}$ before the experiment. The impact velocity of the penetrator mainly depends on the initial charge in the high-pressure chamber. In the present study, we used the compressed air at the pressure from 0.3 MPa to 1.0 MPa, depending on the impact velocity at $130-420 \mathrm{~m} / \mathrm{s}$.

The operation of the ballistic range was as follows: When the quick-action piston valve [7] installed in the high-pressure chamber opens, the shock wave is generated in the shock tube, the film at the downstream end of the acceleration tube is ruptured, and the penetrator model with the sabot starts to move and accelerate in the tube. When the tip of the penetrator reaches the airtight film (Kapton $\left.{ }^{\circledR}\right)$ at the exit of the tube (indicated by "(2)" in Figure 2), it instantaneously tears, and the penetrator goes out of the tube. To avoid the separation between the penetrator and the sabot in the acceleration tube, they were tightly glued together beforehand. The penetrator

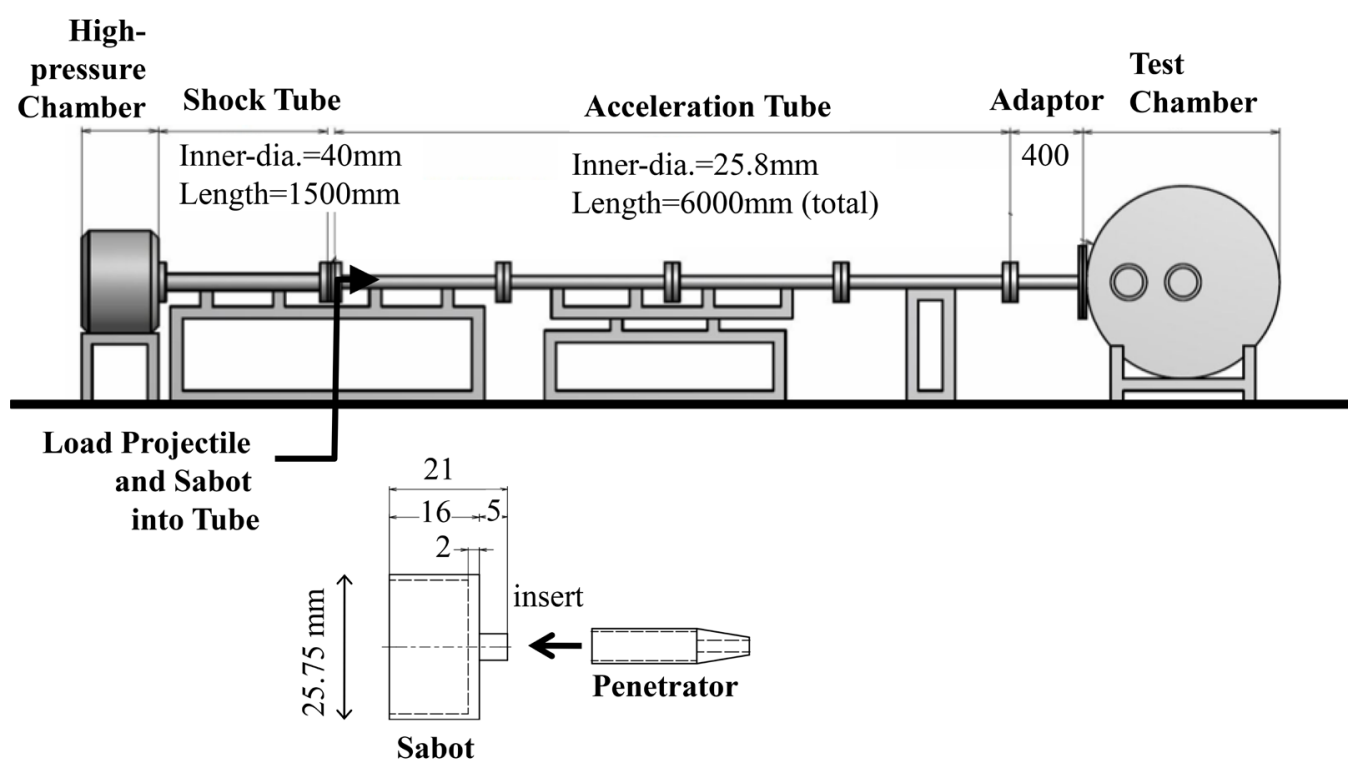

Figure 1. Ballistic range facility and penetrator model with sabot.

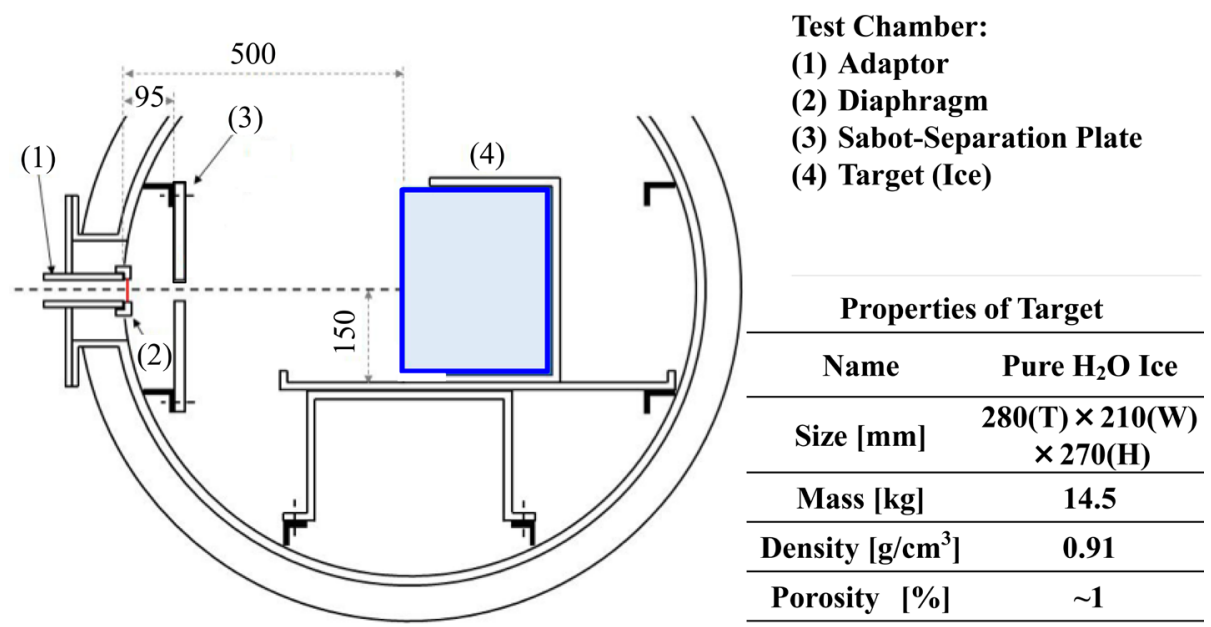

Figure 2. Setup in test chamber. 
is forced to separate from the sabot, when it passes through the sabot-separation plate ("(3)" in Figure 2), which has a hole with a diameter larger than that of the penetrator and smaller than that of the sabot. Figure 3 shows a series of snapshots during the sabot-separation process. In this case, the penetrator was successfully separated from the sabot without receiving significant disturbance in its attitude. In the present method, the necessary impact velocity was obtained by charging the high-pressure chamber appropriately, while the angle of attack at the impact cannot be controlled. In fact, the variation of the attack angle in the experiments was obtained from the disturbance in the attitude of the penetrator at the sabot separation.

The penetrator horizontally hits the target made from water ice. In the present study, the order of the pressure due to the gravitational acceleration was estimated to be $100 \mathrm{~Pa}$, which was obtained by the product of the ice density $\left(\sim 1000 \mathrm{~kg} / \mathrm{m}^{3}\right)$, the gravitational acceleration $\left(\sim 10 \mathrm{~m} / \mathrm{s}^{2}\right)$ and the length scale of the crater $(\sim 0.01 \mathrm{~m})$. The dynamic pressure on the penetrator moving in the ice at the velocity of $200 \mathrm{~m} / \mathrm{s}$ was in the order of $10^{7} \mathrm{~Pa}$. The crushing strength of ice at $273 \mathrm{~K}$ is about $10^{6} \mathrm{~Pa}$ [8]. Consequently, the effect of the gravitational acceleration on the crater forming and the penetration process was negligible, because the pressure due to the gravitational acceleration was much smaller than the others. Of course, the flight of the fragmented ice pieces ejected from the crater was affected by the gravitational acceleration especially in the low-speed regime, and their trajectories were expected to be different from those in the case of the impact experiment conducted in the vertical direction. The size of the target was about $28 \mathrm{~cm}$ (thickness) $\times 21 \mathrm{~cm}$ (width) $\times 27 \mathrm{~cm}$ (height). For the target, we used the commercial pure water ice with specific gravity of $0.90-0.92$ and porosity of about $1 \%$. During the penetration process, the behaviors of the target and the penetrator body were observed through the window of the test chamber. To capture the images of the fragmentation and the crater forming of the ice with high temporal resolution, the high-speed camera (Nobby Tech. Ltd., Phantom Miro ${ }^{\circledR}$ M310) was used. The spatial resolution, frame rate and the exposure time are $512 \times 256$ pixels, 20,000 fps and $4 \mu$ s, respectively. We estimated the velocity and angle of attack (the angle between the body axis and the velocity vector) at the impact from two continuous frames taken from the movie file. The direction of the impact velocity coincided with that of the center line of the acceleration tube. The ice block was carefully set in the test chamber for the center line to be perpendicular

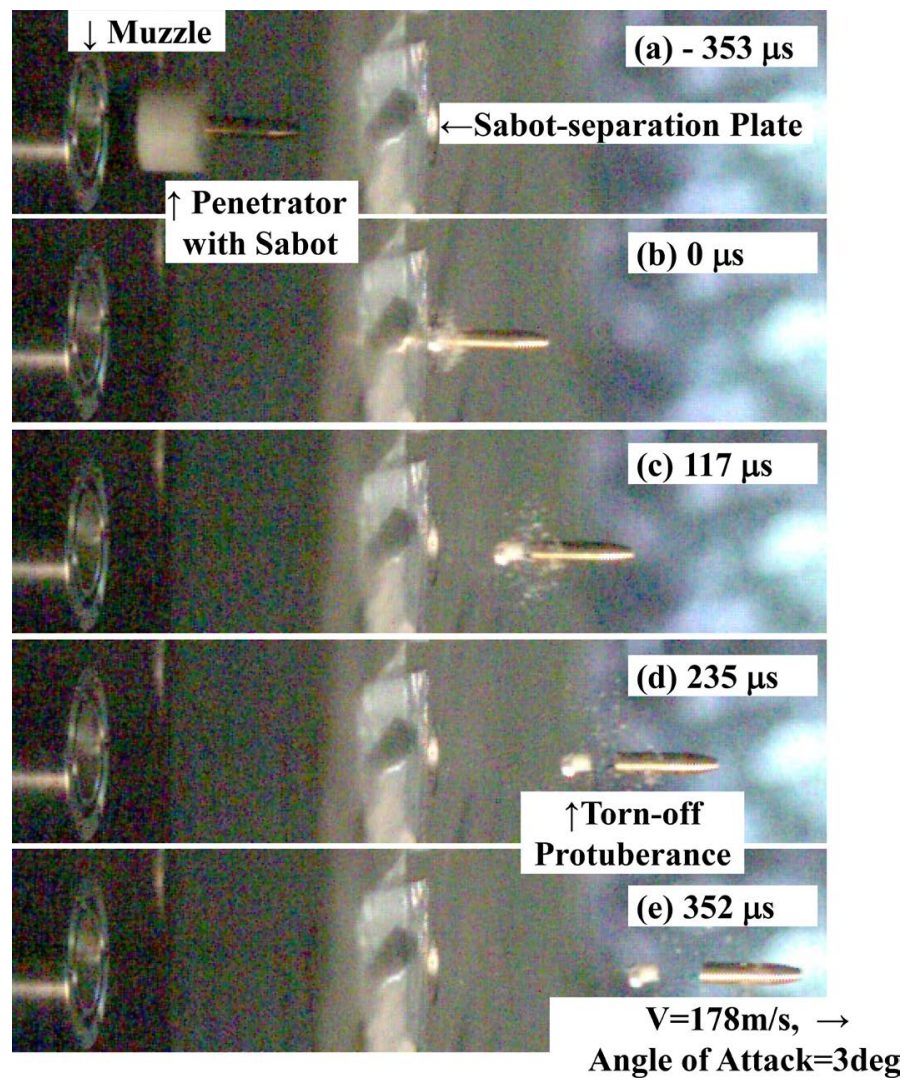

Figure 3. Snapshots of sabot separation process. 
to the surface of the ice. In the spherical coordinate system defined by the velocity vector and the ice surface, the angle of attack was the same as the polar angle. The effect of the azimuth angle was not considered, because the properties of the ice were considered to be almost uniform with respect to the azimuth angle. We defined the two reference points on the body in the frame, that is, the center of the tip and the center of the base. The length scale was obtained by considering that the width of the body in the frame was the same as the diameter of the penetrator. The velocity vector was estimated from the moving distance of the base reference point between the frames. The angle of attack was calculated from the geometrical relation between the velocity vector and the vector connecting the two reference points in the frame. The estimation error mainly depends on the image blur in each frame. It is expected to be in the order of the ratio of the exposure time to the interval between the frames, that is, about $10 \%$ in the present study. In addition, the viewing direction of the camera was not perfectly perpendicular to the velocity vector, and the effect of the perspective viewing increased the estimation error for the angle of attack.

All the experiments were conducted at the room temperature and the atmospheric pressure. It was confirmed that melting did not occur at the surface of the ice before the shot of the ballistic range. However, the crushing strength of ice significantly increases with the decrease in the temperature below $273 \mathrm{~K}$ [8]. The uniformity of the temperature in the ice depended on the temperature in the test chamber and the elapsed time (about $5-10$ minutes) for the setup of the ice in it. To reduce the uncertainty and non-uniformity in the properties of ice, it is necessary to conduct the experiments in the cold room under the temperature control as in [4]-[6].

Six types of the penetrator models shown in Figure 4 were used for the experiments. The body consisted of the cylindrical tube $(6 \mathrm{~mm}$ diameter, $18 \mathrm{~mm}$ length, and $0.5 \mathrm{~mm}$ thickness) and the nose part. The nose shapes were cones (Types A and B), ogives (Types C and D), or inverse-ogives (Types E an F). The shape of the ogive nose was determined after the shape of the LUNAR-A penetrator [3]. Types B, D and F had spherical caps at their tips. For Types A, C and E, the flow-through duct was set with its inlet and outlet at the nose tip and the base of the body, respectively, expecting the relaxation effect on the impact force. In Figure 4, the cross-section of the flow-through duct is shown in grey color with the arrows indicating its inlet and outlet. The penetrator models were made from brass and the mass varied from $2.0 \mathrm{~g}$ to $2.5 \mathrm{~g}$, depending on the model shape. The center of gravity was located $43 \%-52 \%$ of the length from the tip.

\section{Results and Discussion}

\subsection{Observation of Fragmentation of Icy Target and Crater Forming}

Figure 5 shows a series of snapshots taken from the movie file. The penetrator shape was Type E. The velocity

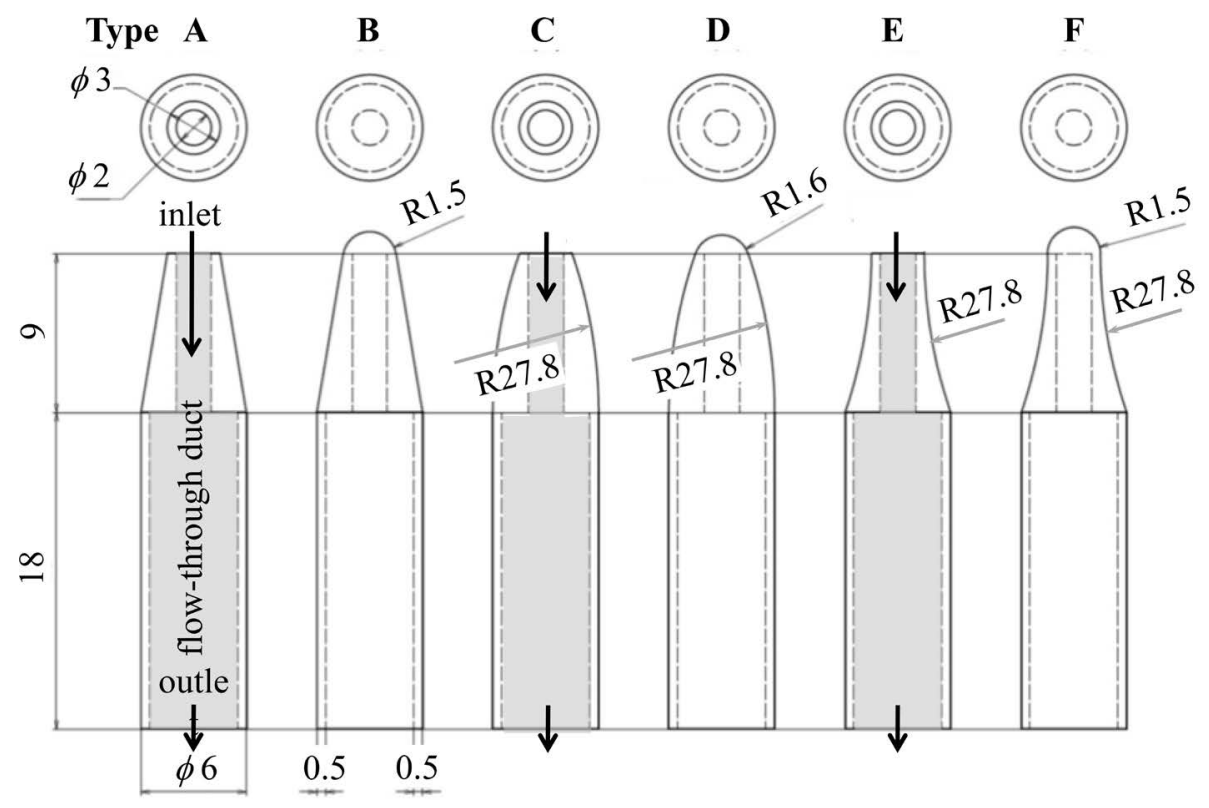

Figure 4. Shapes of penetrator models. 

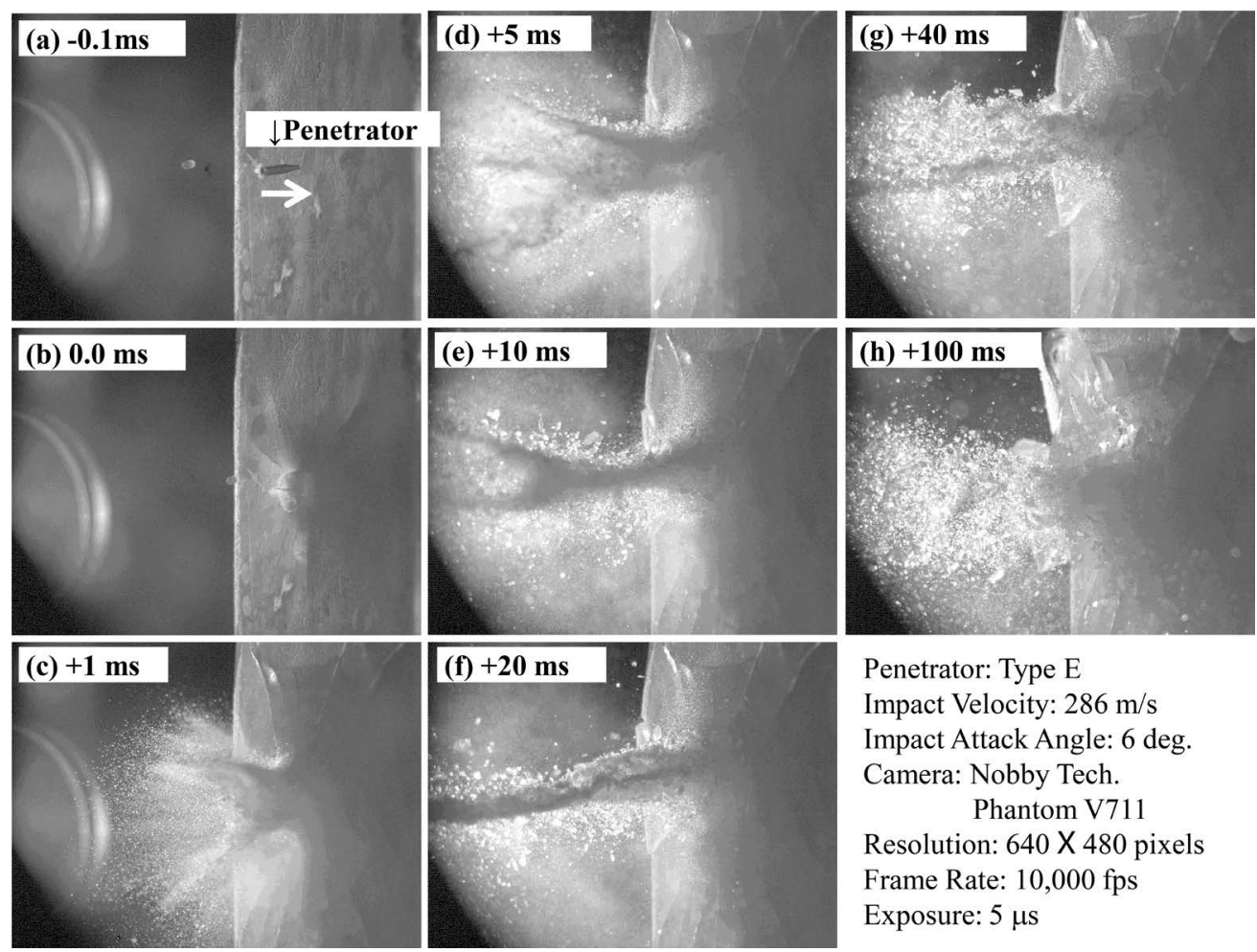

Penetrator: Type E

Impact Velocity: $286 \mathrm{~m} / \mathrm{s}$

Impact Attack Angle: $6 \mathrm{deg}$.

Camera: Nobby Tech.

Phantom V711

Resolution: $640 \times 480$ pixels

Frame Rate: $10,000 \mathrm{fps}$

Exposure: $5 \mu \mathrm{s}$

Figure 5. Jet-like flow of ice pieces crushed by penetrator.

and angle of attack at the impact were $286 \mathrm{~m} / \mathrm{s}$ and 6 degrees, respectively. Only in this case, the high-speed and high-resolution camera (Nobby Tech. Ltd., Phantom ${ }^{\circledR}$ V711) was used. The resolution, frame rate and the exposure time were $640 \times 480$ pixels, $10,000 \mathrm{fps}$ and $5 \mu \mathrm{s}$, respectively. The process of the penetration and the crater forming were clearly visualized in detail as follows: Just after the impact at $0.0 \mathrm{~ms}$, many cracks quickly spread in the radial direction (Figure 5(b)). Simultaneously the fragmented ice pieces were ejected from the impact point and a crown-shaped jet of ice pieces was formed (Figure 5(c)). Such conical-shaped jet continued only for about $5 \mathrm{~ms}$ (Figure 5(d)). The shape of the jet was changed from the conical type to the column type normal to the surface of the target (Figure 5(e)). The normal jet of fragmented ice pieces continued for more than $50 \mathrm{~ms}$ (Figures 5 (f)-(h)). The duration of the normal jet was much longer than that of the crown-shaped jet. From the images of Figure 5(e) and Figure 5(f), the ejecta of the normal jet seems to be composed of fine-grained ice pieces in the inner zone and coarse-grained ice pieces surrounding them. It is expected that such coaxial twozones structure of the jet may be related to the two-stage structure of the crater on the ice, that is, a deep pit and a shallow spall surrounding it, as explained later. The presence of the long-duration normal jet is one of the characteristic features of the fragmentation and crater forming of the icy target.

The presence of the normal jet makes the penetration difficult, because the penetrator tends to be bounced back on the stream of the normal jet without being gripped in the ice. Figure 6 shows the impact at an insufficient velocity for penetration. The penetrator shape was Type B. The velocity and angle of attack at the impact were $126 \mathrm{~m} / \mathrm{s}$ and 6 degrees, respectively. In this case, the penetrator was bounced back from the target as shown in Figure 6(g) and Figure 6(h).

The bounce back also occurs for the impact at a high angle of attack, as shown in Figure 7. The penetrator shape was Type D. The velocity and angle of attack at the impact were $192 \mathrm{~m} / \mathrm{s}$ and 25 degrees, respectively. In case that the penetrator collides with the surface of the target at a large angle of attack, the penetration path is rapidly inclined, and the tail of the body significantly hits the ice, when it is submerged under the surface of the target. In fact, the deformation of the penetrator body was often observed in the rear part after the impact as shown in Figure 7.

In the present study, we tested the penetrator models with the flow-through duct, expecting the relaxation effect of the impact force on the body. Figure 8 shows the close-up views of the behavior of the crushed ice piec- 
es around the tail of the penetrator just after the impact. The penetrator shape was Type E. The velocity and angle of attack at the impact were $290 \mathrm{~m} / \mathrm{s}$ and 5 degrees, respectively. The presence of a small jet from the base indicates that a part of ice pieces, which were produced in front of the penetrator nose, entered by the inlet (see
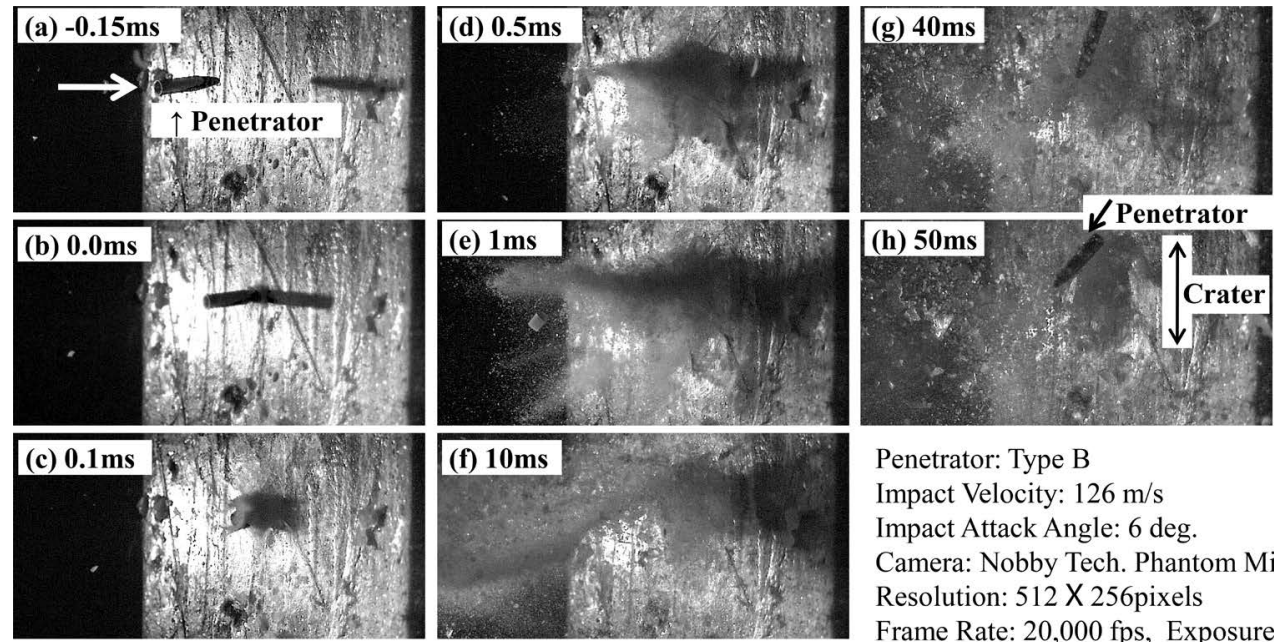

Penetrator: Type B

Impact Velocity: $126 \mathrm{~m} / \mathrm{s}$

Impact Attack Angle: 6 deg.

Camera: Nobby Tech. Phantom Miro M310

Resolution: 512 X 256pixels

Frame Rate: 20,000 fps, Exposure: $4 \mu \mathrm{s}$

Figure 6. Bounce back of penetrator after low speed impact.
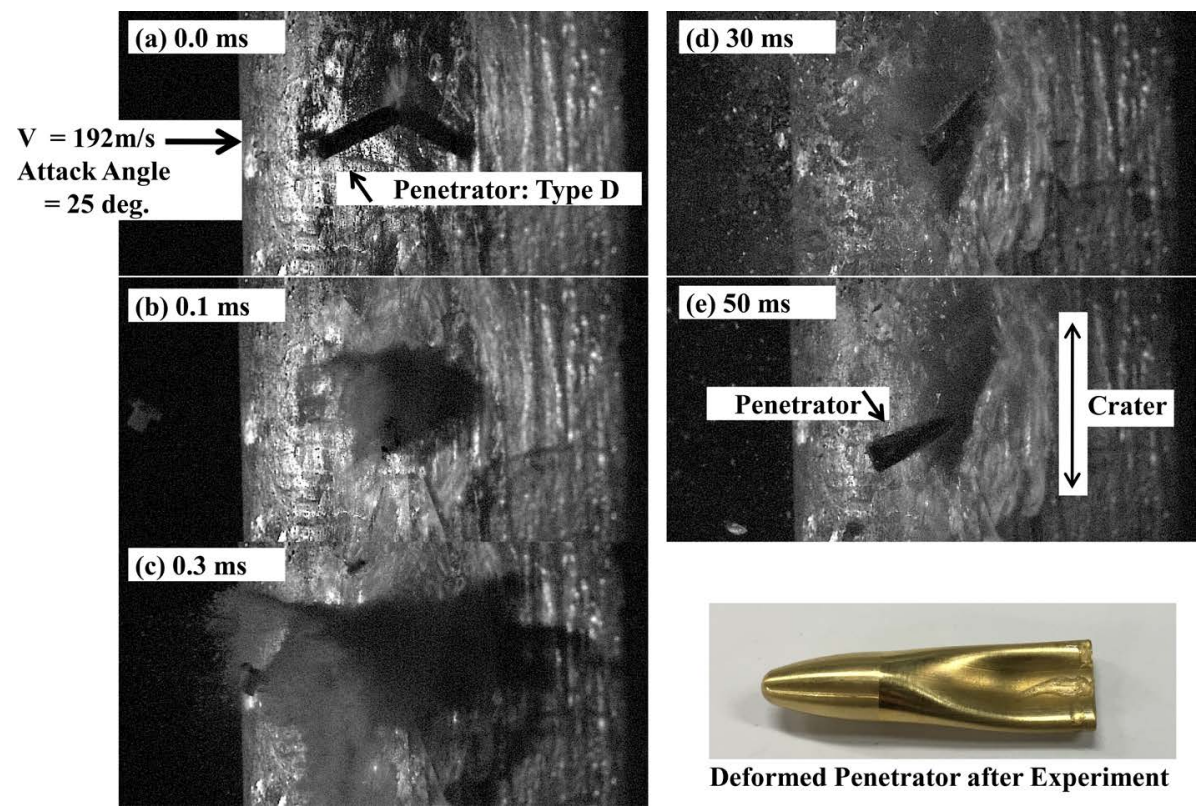

Figure 7. Bounce back of penetrator after high-angle-of-attack impact.

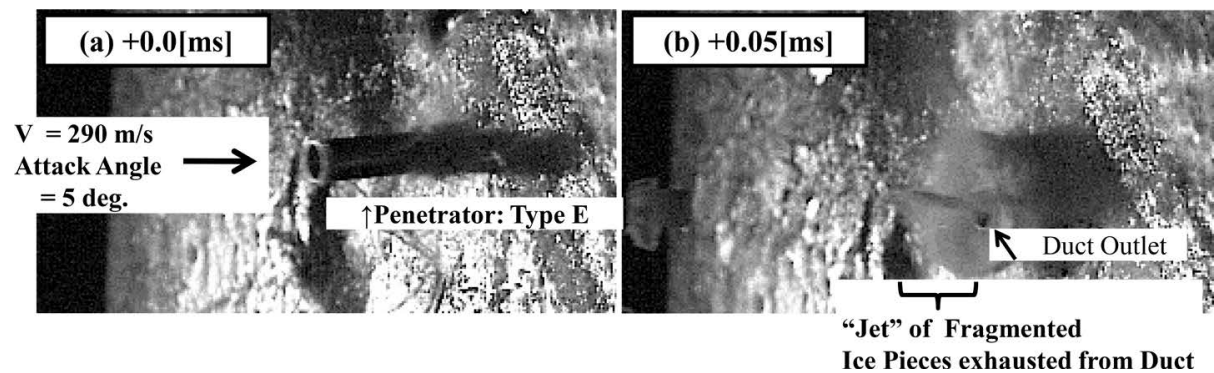

Figure 8. Small jet of crushed ice pieces exhausted from outlet of flow-through duct. 
Figure 4), went through the duct and were finally exhausted from the outlet, forming a small jet. Considering the momentum of this small jet, the relaxation of the impact force on the body is expected. In this case, the penetrator successfully stopped in the target without being bounced back.

\subsection{Observation of Crater Made by Penetrator on Ice}

The final shape of the crater generated by the impact of the penetrator was investigated. Figure 9 shows a typical top view of the crater. The penetrator shape was Type $\mathrm{D}$. The velocity and angle of attack at the impact were $233 \mathrm{~m} / \mathrm{s}$ and 1.7 degrees, respectively. The cracks were formed in the radial direction from the center of the whitish zone covered with fragmented ice pieces. The detailed observation in Figure 10 clarifies that the crater was

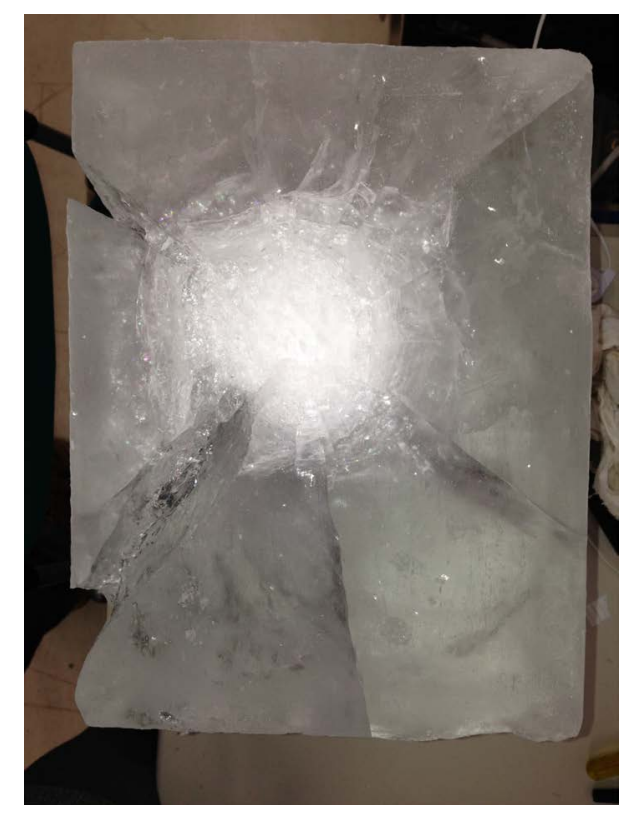

Figure 9. Typical top view of crater on ice.

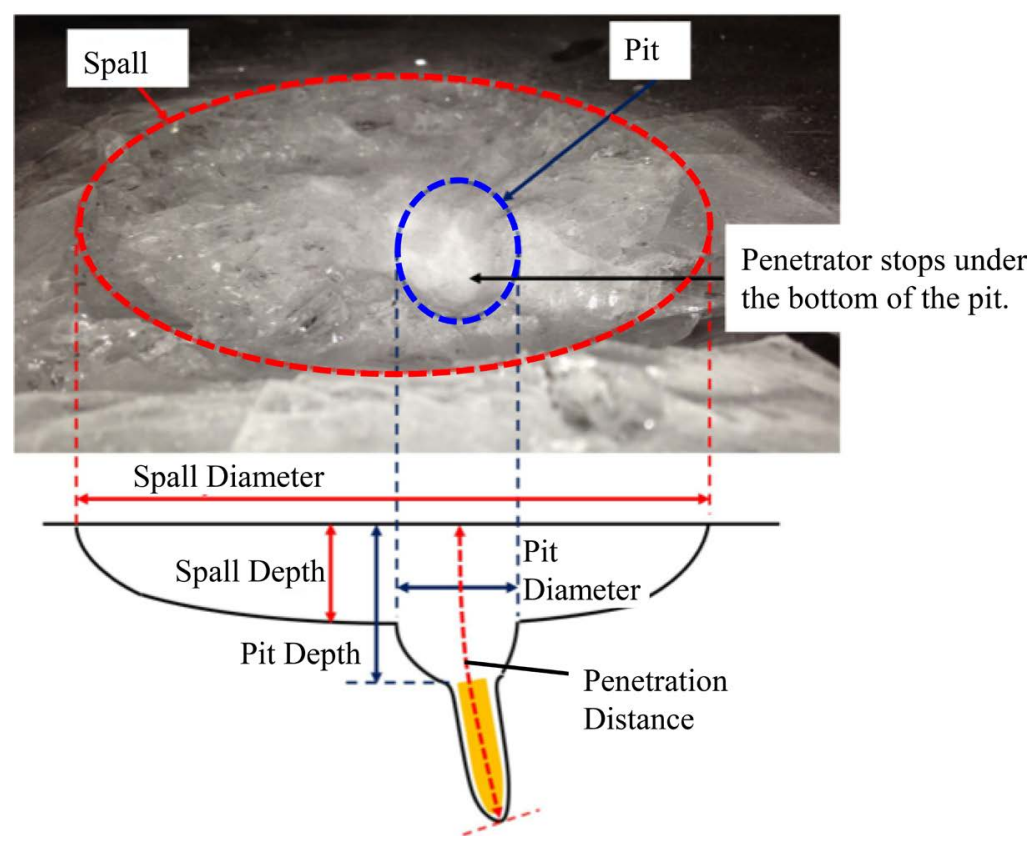

Figure 10. Two-stage and no-rim structure of crater on ice. 
divided into two stages: a deep "pit" in the center and a shallow "spall" around it. In case of successful penetration, the penetrator stopped under the bottom of the pit. The crushed ice pieces were removed from the crater by the jet-like flow from the surface. As a result, a rim, which is a circular hill around a crater, was not formed. When the target was made from the oil clay, the circular accumulation of the clay scraped by the penetrator forms a rim, as shown in Figure 11. Such two-stages and no-rim structure is classified into the "pit" type in [4]. The other types, for example, the tabular plateau type, were not observed in the present study.

The shape of the crater can be visualized by making a mold by pouring plaster into the crater and taking it out after the plaster has been solidified and the ice disappears due to melting [9]. Figure 12 shows a mold of the crater in Figure 10. The two-stage structure is clearly seen. The surface condition of the pit looks different from that of the spall as shown in Figure 13. The surface roughness size in the pit was smaller than that in the spall. The most fine-grained ice pieces were found around the penetrator. Figure 14 shows a block of ice collected after the experiment. In this case, the impact velocity was $423 \mathrm{~m} / \mathrm{s}$ and the target has been completely

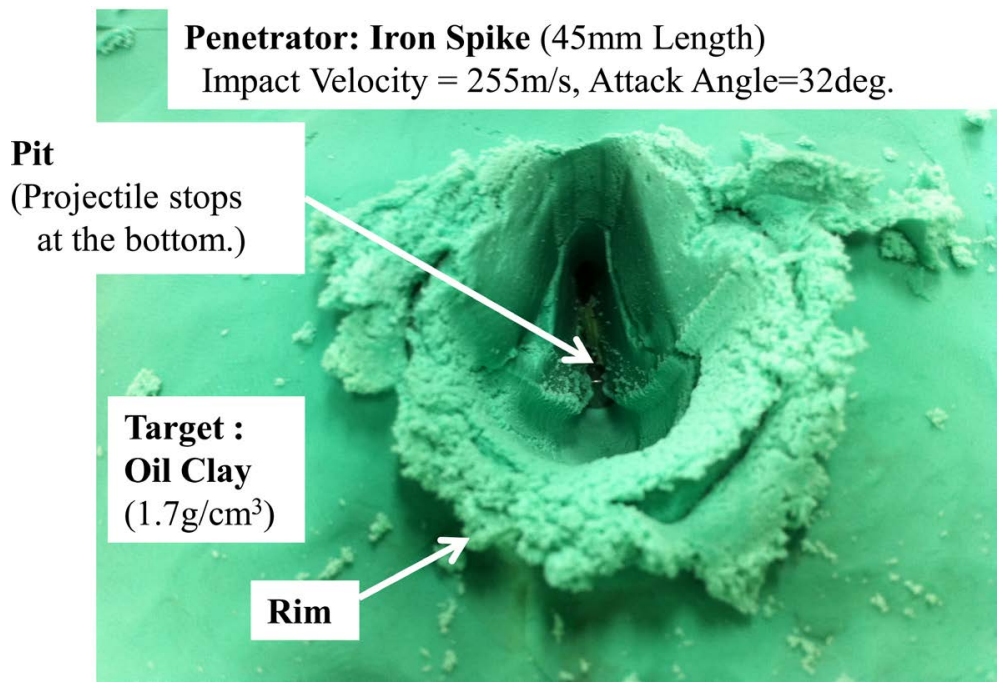

Figure 11. Rim surrounding crater on oil clay target.
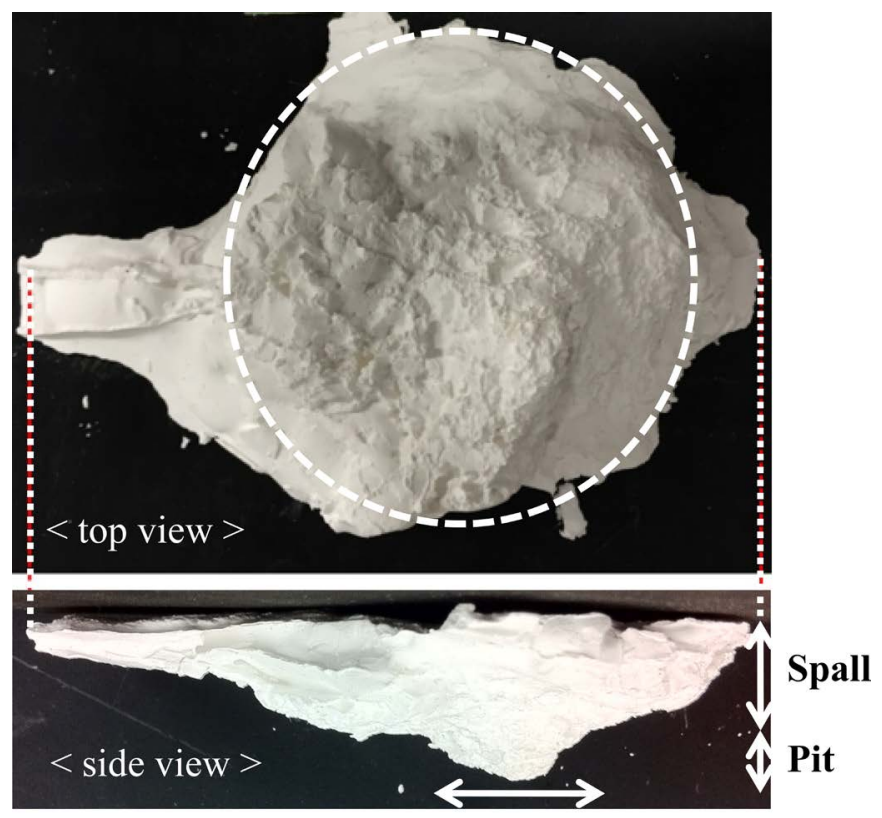

Pit

Figure 12. Visualization of crater by plaster pouring method. 


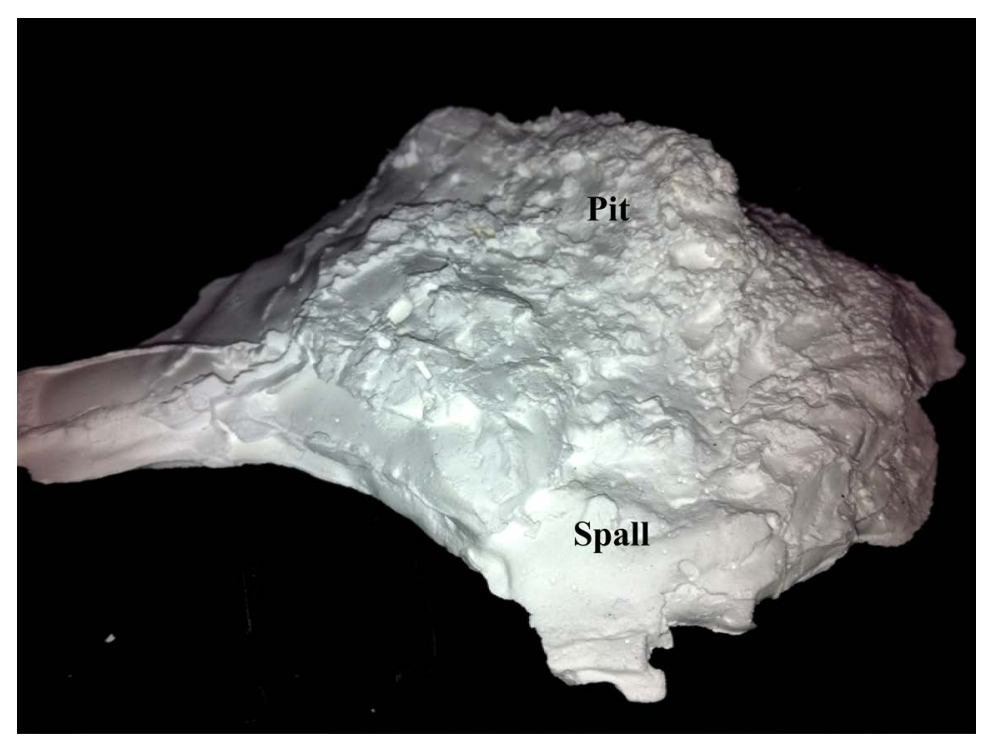

Figure 13. Surface roughness in pit and spall (note: The upper side of the picture corresponds to the bottom of the crater).

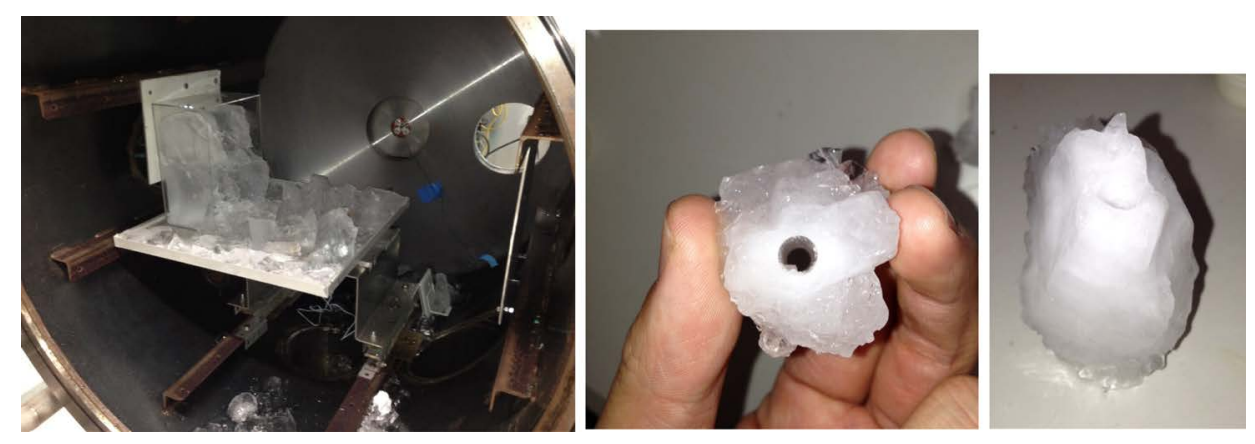

Figure 14. A block of recompressed fine-grained ice pieces and a trace of penetrator in it (left: Destroyed target, Middle: Recompressed ice piece (frontal view), Right: Recompressed ice piece (overall view).

broken into pieces. The hole created in the block was a trace of the penetrator, because its diameter coincided with that of the penetrator. The whitish opaque appearance of the ice block indicated that it was formed by recompression of very-fine-grained ice pieces. The above facts suggest the presence of the cascade process: the spall was formed by scraping out of coarse-grained ice pieces, the pit was formed by that of fine-grained ones, and finally very-fine-grained ones were crushed from fine-grained ones around the penetrator body. Compression by the motion of the penetrator made fragmented ice pieces aggregate into a block of ice as shown in Figure 14. Such features come from the brittle and aggregating nature of ice.

The plaster pouring technique is also useful to visualize the trajectory of the penetrator in the ice. In [9], the analysis model of the penetration dynamics was validated by comparison with the experimental results of the trajectory visualization, the penetration distance and the final attitude of the penetrator. By combining the results obtained by the visualization of the crater formation and the final crater shape, the process of the penetration into ice is summarized as illustrated in Figure 15. It is divided into four steps: cracking, formation of the spall, formation of the pit and the end of penetration.

\subsection{Relation of Crater Diameter and Penetration Distance versus Impact Velocity}

Figure 16 and Figure 17 show the relation of the penetration distance and the spall (crater) diameter, respectively, versus impact velocity. The penetration distance is defined as the distance measured from the impact point to the stop position of the nose tip along the trajectory. In case of successful penetration, the penetrator stopped under the bottom of the pit, as shown in Figure 10. The penetration distance was experimentally estimated as the summation of the body length and the distance between the impact point and the tail of the pene- 


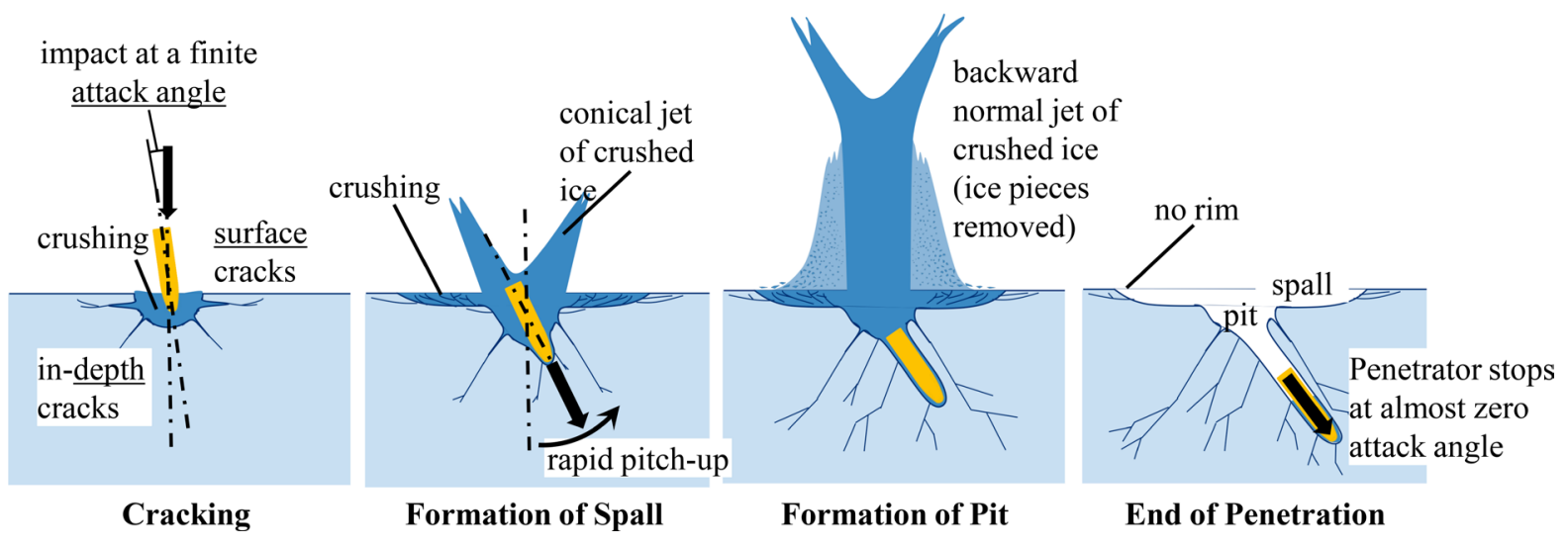

Figure 15. Illustration of penetration process into ice.

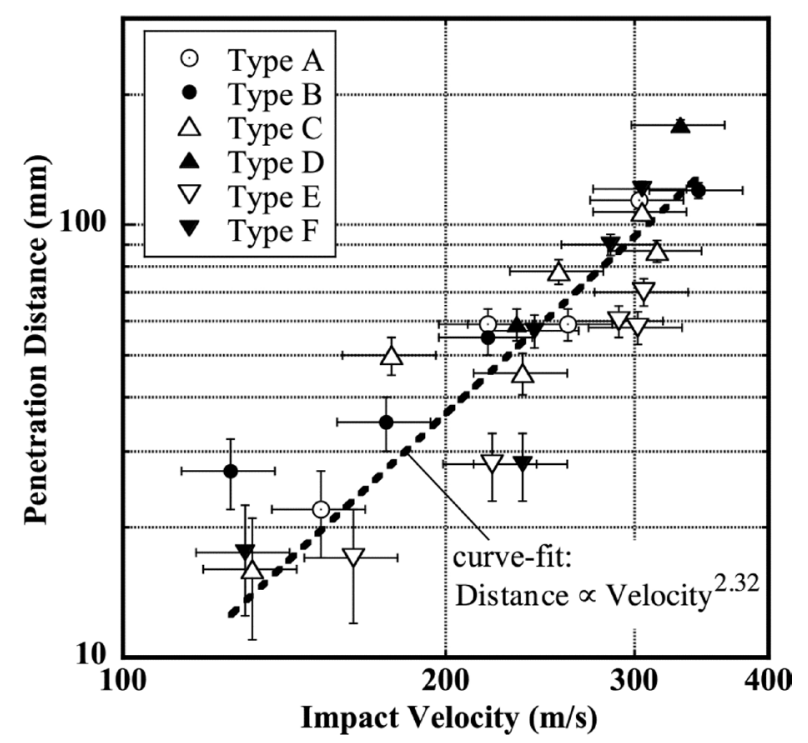

Figure 16. Relation between penetration distance and impact velocity.

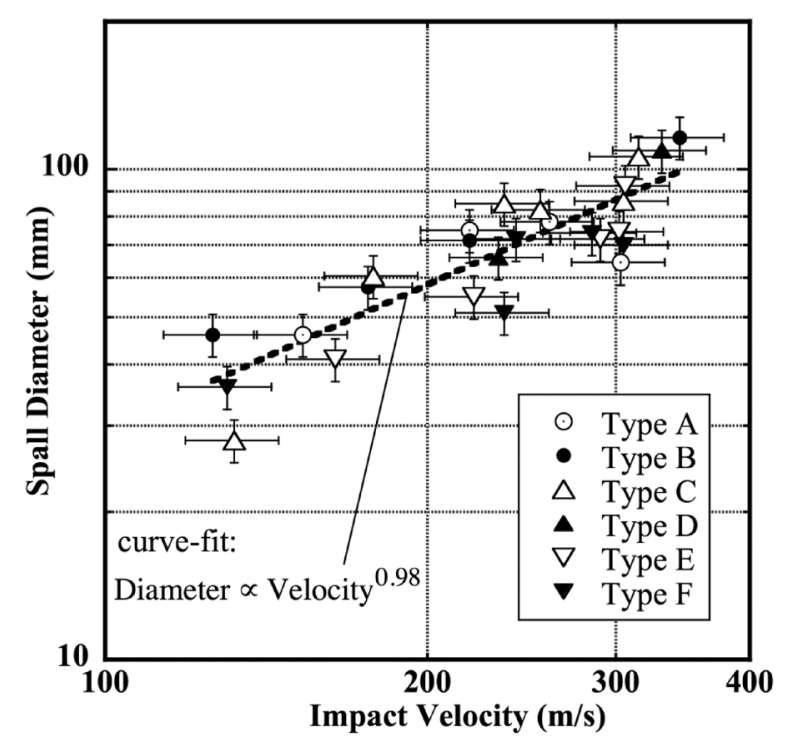

Figure 17. Relation between spall diameter and impact velocity. 
trator. The estimation error was expected to be within $\pm 5 \mathrm{~mm}$. The edge of the spall was not perfectly circular as shown in Figure 12. The diameter was measured assuming an averaged circle with the experimenter's eyes. The measurement error was expected to be $\pm 10 \%$. The estimation error of the impact velocity was $\pm 10 \%$ as discussed in Chapter 2. Then the error bars were obtained as shown in the figures. The dashed lines in the figures indicate the least-square fitting to all the experimental data using the power-law model. The power-law exponent is 2.32 for the penetration distance and 0.98 for the spall diameter.

The power-law exponent of 2.32 for the penetration distance means that it roughly increases with the square of the impact velocity. It implies that the kinetic energy of the penetrator moving at the impact velocity is dissipated by the work done by its motion against the crushing strength of ice for the penetration distance. This is a rough understanding and the penetration distance should be determined after the analysis on the complicated motion of the body in the target, depending on the velocity and angle of attack at the impact, the strength of the target material, the shape, the mass, the moment of inertia and the center-of-gravity location of the penetrator, and so on. In [9], the numerical method to predict the force acting on the penetrator was developed and the penetration distance was predicted after the penetration trajectory analysis.

The power-law exponent of 0.98 for the spall diameter means that it almost linearly increases with the impact velocity. In [4], the relation between the crater dimensions and the late stage effective energy was investigated. In the general scaling law of crater [10], the late stage effective energy is defined as the work done by the pressure behind the shock wave, which has been formed in front of the impactor, to the target material with the volume of the impactor, that is, the product of the pressure rise by the shock wave and the impactor volume. It is known to be effective, when the strength of the target material is dominant for crater formation, and is given by [4]:

$$
\begin{gathered}
E_{L S}=p_{\text {shock }} v o l_{\text {imp }}, \\
p_{\text {shock }}=\frac{1}{2} \xi \rho_{0} V_{\text {impact }}\left(C_{0}+\frac{1}{2} \xi V_{\text {impact }}\right),
\end{gathered}
$$

where $\xi$ is the parameter related to the acoustic impedance matching between the target and impactor materials [11]. When the acoustic impedance (the product of acoustic velocity and density) of the impactor material (brass in the present study) is higher than that of the target material (ice), $\xi$ becomes larger below the maximum allowable value of 2.0 and higher pressure is generated behind the shock wave. In the impact experiments into ice using the cylindrical projectiles with the diameter and length $15 \mathrm{~mm}$ and $10 \mathrm{~mm}$, respectively, the tendency that the spall diameter linearly increases with the late stage effective energy was found irrespective to the material of the impactor [4]. In the present study, the impact velocity was much smaller than the acoustic velocity of ice (about $3000 \mathrm{~m} / \mathrm{s}$ ). From Equations (1) and (2), the late stage effective energy is approximately in proportion to the impact velocity, because $C_{0}$ is dominant in the parentheses of the right hand side of Equation (2). Consequently, the power-law exponent obtained in Figure 17 is consistent with the result in [4]. The above tendency can be explained in a different way by applying the blast wave analogy [12]. Considering that the floor of the spall was relatively flat as illustrated in Figure 10, it seems reasonable that the spall was formed by the cylindrical blast wave propagating in the ice near its surface rather than the spherical wave. From the blast wave analogy, the pressure behind the cylindrical blast wave changes with the inverse of the square of the propagation distance $r$ :

$$
p_{c b w} \propto E / r^{2},
$$

where $E$ is the released energy per unit length. In the blast wave analogy; $E$ is the same as the drag force acting on the nose of the body and is in proportion to the square of the impact velocity. Considering that crushing of ice does not occur, when $p_{c b w}$ becomes smaller than the crushing strength of ice $S_{c}$, the spall radius $r_{s}$ satisfies the relation:

$$
r_{s} \propto\left(E / S_{c}\right)^{1 / 2} .
$$

Assuming that $S_{c}$ is constant, it is deduced that the spall diameter linearly increases with the impact velocity.

The uncertainty of the experimental data is expected not to be small. It was mainly caused by the non-uniformity of the properties of the ice target, the variation of the temperature environment in the test chamber and the irregularity in the crack formation and the behavior of the fragmented ice pieces. It is hard to eva- 
luate the degree of uncertainty at present. To reduce it, the number of the experimental data should be significantly increased and the statistical data processing is necessary.

\section{Conclusion}

The penetration of the impact probe into the icy target was experimentally simulated by the ballistic range. Cylindrical slender bodies with various nose shapes were tested. The process of the penetration and crater forming in the ice was clearly visualized by the high-speed video camera with high temporal resolution at 20,000 fps. The backward normal jet of crushed ice pieces was observed for more than $50 \mathrm{~ms}$ after the formation of the crown-shaped jet of ice pieces for much shorter duration of about $5 \mathrm{~ms}$. The impact at a low velocity or a high angle-of-attack results in the bounce back of the penetrator from the target on the backward stream of ice pieces. The characteristic features of the crater shape on the icy target were observed, and that is, the surface and in-depth cracks spreading from the impact point, the deep pit and the shallow spall surrounding the pit. In case of successful penetration, the penetrator stopped under the bottom of the pit. No rim (circular hill) was formed around the crater. Observation of the crater surface and ice pieces recovered after the experiment suggests the cascade fragmentation process from larger ice pieces to fine-grained ones and the recompression into aggregated ice blocks. The penetration distance roughly increases with the square of the impact velocity. On the other hand, the spall (crater) diameter almost linearly increases with the impact velocity. That tendency is consistent with the existing scaling laws for the crater dimensions at low impact velocities relative to the acoustic velocity of the target material. When the penetrator has a flow-through duct from the tip to the base of the body, it was observed that crushed ice pieces entering the inlet were exhausted from the outlet. The momentum of the exhausted flow of ice pieces is expected to result in the relaxation of the impact force on the body. The results obtained in the present study will be useful to determine the guideline to design a penetrator into an icy target for explorations of water-containing objects in the solar system.

\section{Acknowledgements}

This work has been supported by Grant-in-Aid for Scientific Research (B) No. 25289301 of Japan Society for the Promotion of Science. The trial use of the high-resolution high-speed camera Phantom ${ }^{\circledR}$ V711 was provided by Nobby Tech. Ltd.

\section{References}

[1] Gilmour, I. and Sephton, M.A. (2003) An Introduction to Astrobiology. Cambridge University Press, Cambridge.

[2] Lorenz, R.D. (2011) Planetary Penetrators: Their Origins, History and Future. Advances in Space Research, 48, 403431. http://dx.doi.org/10.1016/j.asr.2011.03.033

[3] Shiraishi, H., Tanaka, S., Hayakawa, M., Fujimura, A., Mizutani, H. and Suzuki, K. (2006) Dynamical Characteristics of a Planetary Penetrator. Penetrometry in the Solar System II, Graz, 25-28 September 2006, 217-232.

[4] Kato, M., Iijima, Y., Arakawa, M., Okimura, Y., Fujimura, A., Maeno, N. and Mizutani, H. (1995) Ice-on-Ice Impact Experiments. ICARUS, 113, 423-441. http://dx.doi.org/10.1006/icar.1995.1032

[5] Iijima, Y., Kato, M., Arakawa, M., Maeno, N., Fujimura, A. and Mizutani, H. (1995) Cratering Experiments on Ice: Dependence of Crater Formation on Projectile Materials and Scaling Parameter. Geophysical Research Letters, 22, 2005-2008. http://dx.doi.org/10.1029/95GL01894

[6] Arakawa, M., Shirai, K. and Kato, M. (2000) Shock Wave and Fracture Propagation in Water Ice by High Velocity Impact. Geophysical Research Letters, 27, 305-308. http://dx.doi.org/10.1029/1999GL010841

[7] Oguchi, H., Funabiki, K. and Sato, S. (1974) An Experiment on the Shock Tube with a Mechanical Shock Valve. Bulletin of Institute of Space and Astronautical Science, University of Tokyo, 10, 778-786. (In Japanese)

[8] Hobbs, P.V. (1974) Ice Physics. Oxford University Press, Oxford.

[9] Suzuki, K., Numba, K. and Watanabe, Y. (2015) Study on Dynamics of Penetrator into Ice. Web Paper Archives of the 30 th International Symposium on Space Technology and Science, Kobe, 4-10 July 2015, 2015-k-21. http://archive.ists.or.jp/upload_pdf/2015-k-21.pdf

[10] Mizutani, H., Kawakami, S., Takagi, Y., Kato, M. and Kumazawa, M. (1983) Cratering Experiments in Sands and a Trial for General Scaling Law. Journal of Geophysical Research, 88, A835-A845. http://dx.doi.org/10.1029/JB088iS02p0A835

[11] Mizutani, H., Takagi, Y. and Kawakami, S. (1990) New Scaling Laws on Impact Fragmentation. ICARUS, 87, 
307-326. http://dx.doi.org/10.1016/0019-1035(90)90136-W

[12] Anderson Jr., J.D. (1989) Hypersonic and High Temperature Gas Dynamics. McGraw-Hill, New York.

\section{Nomenclature}

$C_{0}$ : acoustic velocity of target material

$E$ : released energy of impact per unit length

$E_{L S}$ : late stage effective energy

$p_{c b w}$ : pressure behind cylindrical blast wave

$p_{\text {shock}}$ : pressure behind shock wave

$r$ : propagation distance of blast wave

$r_{s}$ : spall radius

$S_{c}$ : crushing strength of ice

$V_{\text {impact }}$ impact velocity

$v_{\text {o }} l_{\text {imp }}$ : volume of penetrator

$\rho_{0}$ : initial density of target material

$\xi$ : acoustic impedance matching parameter 